$h$ is uniformly approximable on $\tilde{\chi}_{1 / m}(K)$ by rational functions of $z$, because $d x d y\left(\tilde{\chi}_{1 / m}(K)\right)=0$. If $h^{\prime} \in R\left(\tilde{\chi}_{1 / m}(K)\right)$, such that $\max \left|h-h^{\prime}\right|<\varepsilon / 2$, then $\max \left|f-h^{\prime} \circ \tilde{\chi}_{1 / m}\right|<\varepsilon$, so that $f \in R_{G}(K)$.

In particular every set $K \subset G$ is rationally convex. Every such set is also polynomially convex (note that if $g_{0} \in G \backslash K$, the function

$$
h(\lambda, g)=\sum_{n=1}^{\infty} \frac{1}{2^{n+2}}\left(1+\chi_{p_{n}}\left(g_{0}\right) \tilde{\chi}_{p_{n}}(\lambda, g)\right)
$$

admits the following conditions:

$$
h \in A_{G}, \quad h\left(\lambda_{0}, g_{0}\right)=1 \quad \text { and } \quad|h| \underset{G \backslash\left\{g_{0}\right\}}{<1} .
$$

\title{
References
}

[1] R. Arens and I. Singer, Generalized analytic functions, Trans. Amer. Math. Soc. 81 (1956), 379-393.

[2] T. W. G a m e $1 \mathrm{i}$ n, Uniform algebras, Prentice-Hall, N. J., 1969.

$$
\begin{aligned}
& \text { Presented to the semester } \\
& \text { Spectral Theory } \\
& \text { September 23-December 16, } 1977
\end{aligned}
$$

BANACH CENTER PUBLICATIONS, VOLUMB 8

PWN-POLISH SCIENTIFIC PUBLISHERS
WARSAW 1982

\section{A MULTI-DIMENSIONAL SPECTRAL THEORY IN $C^{*}$-ALGEBRAS}

\author{
F.-H. VASILESCU
}

Department of Mathematics, The National Institute for Scientific and Technical Creation, Bucharest, România

\section{Preliminaries}

The aim of this work is to introduce a notion of joint spectrum for finite commuting systems of elements of (not necessarily commutative) $C^{*}$-algebras and to present some significant relevant results. Our definition originates in a characterization of the joint spectrum (in the sense of J. L. Taylor [9]), which can be stated for commuting systems of operators in Hilbert spaces [11]. The present notion of joint spectrum is intrinsically connected to a given $C^{*}$-algebra but its general properties can be easily derived from suitable representations on Hilbert spaces. We intend to carry out the whole programme of J. L. Taylor concerning the properties of the joint spectrum and the construction of the analytic functional calculus [9], [10]. Since we work with more restrictive conditions, there occur considerable simplifications of most of the proofs, in comparison with those of Taylor's. However, the analytic functional calculus will be given by a canonical formula, extending the classical formula of Martinelli for analytic functions in several variables [6], [7]. The results related to the commuting systems of operators on Hilbert spaces, which form the basic part of this work, are exposed from [11], [14] and [13]. The terminology and facts concerning $C^{*}$-algebras can be found in [4] and [8].

From now on $s=\left(s_{1}, \ldots, s_{n}\right)$ will denote a fixed system of indeterminates (nevertheless, the index $n$ may vary). Let $\Lambda[s]$ be the exterior algebra over the complex field generated by $s_{1}, \ldots, s_{n}$. For any integer $p, 0 \leqslant p \leqslant n$, we denote by $\Lambda^{p}[s]$ the space of all homogeneous exterior $p$-forms in $s_{1}, \ldots, s_{n}$. Of course, $\Lambda^{0}[s]$ is identified with the complex field $C$. Every space $\Lambda^{p}[s]$ has a natural Hilbert space structure, in which the elements

$$
s_{j_{1}} \wedge \ldots \wedge s_{j_{p}} \quad\left(1 \leqslant j_{1}<\ldots<j_{p} \leqslant n\right)
$$

form an orthogonal basis. The Hilbert space structure of $\Lambda[s]$ is induced by the formula

$$
\Lambda(s)=\bigoplus_{p=0}^{n} \Lambda^{p}[s] .
$$


Consider now the operators

$$
S_{j} \xi=s_{j} \wedge \xi \quad(\xi \in \Lambda[s] ; j=1, \ldots, n) .
$$

The anticommuting system $\sigma=\left(S_{1}, \ldots, S_{n}\right)$ will play an important role in the sequel. Notice that the adjoint of (1.1) is given by

$$
S_{j}^{*}\left(\xi_{j}^{\prime}+s_{j} \wedge \xi_{j}^{\prime \prime}\right)=\xi_{j}^{\prime \prime} \quad(j=1, \ldots, n),
$$

where $\xi_{j}^{\prime}+s_{j} \wedge \xi_{j}^{\prime \prime}$ is the canonical decomposition of an arbitrary element $\xi \in \Lambda[s]$, with $\xi_{j}^{\prime}$ and $\xi_{j}^{\prime \prime}$ not containing $s_{j}$.

From (1.1) and (1.2) we obtain readily the relations

$$
S_{j} S_{k}^{*}+S_{k}^{*} S_{j}= \begin{cases}0, & j \neq k \\ 1, & j=k\end{cases}
$$

The next result is well known in theoretical physics.

LEMMA 1.1. Define $T_{j}=S_{j}$ for $1 \leqslant j \leqslant n$ and $T_{j}=S_{j-n}^{*}$ for $n+1 \leqslant j \leqslant 2 n$. Then the set of monomials of the form

$$
T_{j_{1}} \ldots T_{j_{p}} \quad\left(1 \leqslant j_{1}<\ldots<j_{p} \leqslant 2 n\right)
$$

and the identity form a basis for the space of all linear operators on $\Lambda[s]$.

Proof. Notice that the dimension of the space $A[s]$ is $2^{n}$ and we have exactly $2^{2 n}$ monomials of the prescribed form. Thus it will be sufficient to prove that these monomials generate the space. We use induction with respect to $n$. If $n=1$, the system $\left(1, s_{1}\right)$ is a basis in $A[s]$. Then we have

$$
S_{1}=\left(\begin{array}{ll}
0 & 0 \\
1 & 0
\end{array}\right), \quad S_{1}^{*}=\left(\begin{array}{ll}
0 & 1 \\
0 & 0
\end{array}\right), \quad S_{1} S_{1}^{*}=\left(\begin{array}{ll}
0 & 0 \\
0 & 1
\end{array}\right),
$$

with respect to $\left(1, s_{1}\right)$. It is clear that every $2 \times 2$ matrix is a linear combination of $S_{1}, S_{1}^{*}, S_{1} S_{1}^{*}$ and the identity, hence the assertion is true in this case.

Assume now that the assertion is true up to the order $n$. If $s^{\prime}=\left(s_{1}, \ldots, s_{n}, s_{n+1}\right)$, we have

$$
\Lambda\left[s^{\prime}\right]=\Lambda[s] \oplus S_{n+1} \Lambda[s] .
$$

Note that $P=S_{n+1}^{*} S_{n+1}$ is the projection of $\Lambda\left[s^{\prime}\right]$ onto $\Lambda[s]$ and that $Q=1-P$ $=S_{n+1} S_{n+1}^{*}$, by (1.3). If $V$ is an arbitrary operator on $\Lambda\left[s^{\prime}\right]$, then $V=P V P+$ $+Q V P+P V Q+Q V Q$ and each term of this sum may be represented as desired, by the induction hypothesis.

Let us denote by $M[s]$ the set of all linear operators on the space $A[s]$. We then have the following

COROLlary 1.2. The $C^{*}$-algebra with identity generated by the system $\sigma$ $=\left(S_{1}, \ldots, S_{n}\right)$ is equal to $M[s]$.

Let us now take a $C^{*}$-algebra $B$ with identity, always denoted by 1 . Then the tensor product

$$
M[s, B]=B \otimes M[s]
$$

can be endowed with a cross-norm, which is a $C^{*}$-norm, making it a $C^{*}$-algebra [8]. We identify the algebra $B$ with the subalgebra $B \otimes 1$ of $M[s, B]$ and the algebra $M[s]$ with the subalgebra $1 \otimes M[s]$. The symbol " $\otimes$ " will be also omitted when representing elements of $M[s, B]$.

Consider a commuting system $b=\left(b_{1}, \ldots, b_{n}\right) \subset B$. This system can be associated with the element

with the property

$$
\delta_{b}=b_{1} S_{1}+\ldots+b_{n} S_{n} \in M[s, B]
$$

$$
\delta_{b}^{2}=\sum_{j<k}\left(b_{j} b_{k}-b_{k} b_{j}\right) S_{j} S_{k}=0,
$$

on account of the commutativity of $\left(b_{1}, \ldots, b_{n}\right)$.

Definition 1.3. The commuting system $b=\left(b_{1}, \ldots, b_{n}\right)$ in $B$ is said to be nonsingular if the element

$$
R(b)=\delta_{b}+\delta_{b}^{*}
$$

is invertible in $M[s, B]$. Otherwise, the system $b$ will be called singular.

The set of all points $z=\left(z_{1}, \ldots, z_{n}\right) \in C^{n}$ such that $z-b=\left(z_{1}-b_{1}, \ldots, z_{n}-b_{n}\right)$ is singular will be called the (joint) spectrum of $b$ and will be denoted by $\sigma_{B}(b)$.

We now show that Definition 1.3 is a reasonable extension of the one-dimensional case.

PROPOSITION 1.4. If $b$ is the singleton $\left(b_{1}\right)$ then $b$ is nonsingular if and only if $b_{1}$ is invertible.

Proof. If $b_{1}$ is invertible then $b_{1}^{*-1} S_{1}+b_{1}^{-1} S_{1}^{*}$ is the inverse of $b_{1} S_{1}+b_{1}^{*} S_{1}^{*}$, hence $b$ is nonsingular.

Conversely, by Lemma 1.1 the system $\left(1, S_{1}, S_{1}^{*}, S_{1} S_{1}^{*}\right)$ is a basis in $M[s]$, where $s=\left(s_{1}\right)$. If the element

$$
a_{0}+a_{1}^{\prime} S_{1}+a_{1}^{\prime \prime} S_{1}^{*}+a_{2} S_{1} S_{1}^{*} \in M[s, B]
$$

is the inverse of the self-adjoint element $b_{1} S_{1}+b_{1}^{*} S_{1}^{*}$, then $a_{0}=a_{0}^{*}, a_{1}^{\prime *}=a_{1}^{\prime \prime}$ and $a_{2}^{*}=a_{2}$. Moreover, we must have $b_{1} a_{0}=0, b_{1}^{*}\left(a_{0}+a_{2}\right)=0, b_{1}^{*} a_{1}=1, b_{1} a_{1}^{*}$ $=b_{1}^{*} a_{1}$ and $a_{1} b_{1}^{*}=a_{1}^{*} b_{1}$, with $a_{1}=a_{1}^{\prime}$, whence $a_{1}^{*}=b_{1}^{-1}$ and $a_{0}=a_{2}=0$. In particular, $b_{1}$ is invertible in $B$.

Note that from Proposition 1.4 one infers that $\sigma_{B}(b)=\sigma_{B}\left(b_{1}\right)$, where the last symbol denotes the usual spectrum of the element $b_{1} \in B$.

The next sections will present some other reasons indicating that the above definition of the joint spectrum is legitimate.

\section{The elementary properties of the joint spectrum}

In this section $B$ will be a fixed $C^{*}$-algebra with identity. All *-representations (*-homomorphisms) will be supposed to preserve the identity. 
LEMMA 2.1. If $B^{\prime}$ is another $C^{*}$-algebra with identity and $\varphi: B \rightarrow B^{\prime}$ is a ${ }^{*}$-homomorphism, then for any commuting system $b=\left(b_{1}, \ldots, b_{n}\right)$ in $B$ we have the inclusion

$$
\sigma_{B^{\prime}}(\varphi(b)) \subset \sigma_{B}(b) .
$$

If $\varphi$ is $a^{*}$-isomorphism, then this inclusion is an equality.

Proof. The assertion follows easily from the fact that $\varphi \otimes 1$ is a *-homomorphism of $M[s, B]$ into $M\left[s, B^{\prime}\right]$.

For an arbitrary Hilbert space $X$ we denote by $\mathscr{L}(X)$ the algebra of all bounded linear operators acting on $X$.

We recall that for the $C^{*}$-algebra $B$ there exists, by the Gelfand-Naimark representation theorem [4], [8], a Hilbert space $X$ and an isometric *-isomorphism $\varphi$ of $B$ into $\mathscr{L}(X)$. Then the map $\varphi \otimes 1$ is a *-isomorphism of $M[s, B]$ onto $M[s, \varphi(B)] \subset \mathscr{L}(\Lambda[s, X])$, where $\Lambda[s, X]=X \otimes \Lambda[s]$ (this notation will be kept for arbitrary systems of indeterminates and linear spaces). Although the space $X$ is not uniquely determined by $B$, Lemma 2.1 implies that the commuting systems in $B$ have equal spectra with the corresponding commuting systems in $\varphi(B)$. Therefore there is no essential loss of generality in supposing that $B \subset \mathscr{L}(X)$, where $X$ is a fixed Hilbert space.

If $b=\left(b_{1}, \ldots, b_{n}\right) \subset B$ is a commuting system, then the map $\delta_{b}$, defined on $\Lambda[s, X]$, has the property $\delta_{b}^{2}=0$, whence $\mathscr{R}\left(\delta_{b}\right) \subset \mathscr{K}\left(\delta_{b}\right)$, where $\mathscr{R}$ and $\mathscr{K}$ stand for the range and the kernel, respectively.

Proposition 2.2. Assume that $b=\left(b_{1}, \ldots, b_{n}\right) \subset B \subset \mathscr{L}(X)$ is a commuting system. The system $b$ is nonsingular if and only if $\mathscr{R}\left(\delta_{b}\right)$ is equal to $\mathscr{K}\left(\delta_{b}\right)$.

Proof. This assertion will follow from a more general statement.

LEMMA 2.3. If $a \in \mathscr{L}(X)$ has the property $a^{2}=0$, then $a+a^{*}$ is invertible in $\mathscr{L}(X)$ if and only if $\mathscr{R}(a)=\mathscr{K}(a)$.

Proof of Lemma 2.3. Suppose that $a+a^{*}$ is invertible and denote by $p$ the projection of $X$ onto $\mathscr{K}(a)$. If $y \in \mathscr{K}(a)$ is arbitrary, we have

$$
y=p y=\left(a+a^{*}\right)\left(a+a^{*}\right)^{-1} y=a(1-p)\left(a+a^{*}\right)^{-1} y
$$

since $1-p$ is the projection onto $\overline{\mathscr{R}\left(a^{*}\right)}$, we see that $\mathscr{R}(a)=\mathscr{K}(a)$.

Conversely, the equality $\mathscr{R}(a)=\mathscr{K}(a)$ implies the equality $\mathscr{R}\left(a^{*}\right)=\mathscr{K}\left(a^{*}\right)$; thus, from the decomposition $X=\mathscr{K}(a) \oplus \mathscr{R}\left(a^{*}\right)$ we obtain that $a$ maps $\mathscr{K}\left(a^{*}\right)$ isomorphically onto $\mathscr{K}(a)$ and $a^{*}$ maps $\mathscr{K}(a)$.isomorphically onto $\mathscr{K}\left(a^{*}\right)$. We hence derive that $a+a^{*}$ has a continuous inverse in $\mathscr{L}(X)$.

The proof of Proposition 2.2 follows from Lemma 2.3, applied to $\delta_{b}$ on $A[s, X]$. The only thing to be noted is that if $\delta_{b}+\delta_{b}^{*}$ is invertible in $\mathscr{L}(\Lambda[s, X])$, then it is invertible in $M[s, B]$ as well, since any $C^{*}$-algebra of this type is inverse-closed [4].

Remark 2.4. Proposition 2.2 remains true if we take instead of $X$ a finite direct sum of copies of $X$, which is again a left $B$-module, in a natural way.

For commuting systems $a=\left(a_{1}, \ldots, a_{n}\right) \subset \mathscr{L}(X)$ we denote by $\sigma(a, X)$ the set $\sigma_{A}(a)$, where $A$ is the $C^{*}$-algebra with unit generated in $\mathscr{L}(X)$ by $a_{1}, \ldots, a_{n}$.
This set can be defined only in terms of $X$, as one can see from Proposition 2.2; such being the original definition for the joint spectrum of commuting systems of operators [9], [11].

LEMMA 2.5. Consider a commuting system $b=\left(b_{1}, \ldots, b_{n}\right) \subset B \subset \mathscr{L}(X)$. If $a$ $=\left(a_{1}, \ldots, a_{n}\right)$ is a system in the commutant of $b$ in $\mathscr{L}(X)$ with the property $a_{1} b_{1}+\ldots$ $\ldots+a_{n} b_{n}=1$, then $b$ is nonsingular.

Proof. Notice that

$$
\delta_{b} \delta_{a^{*}}^{*}+\delta_{a^{*}}^{*} \delta_{b}=\sum_{j, k} b_{j} a_{k}\left(S_{j} S_{k}^{*}+S_{k} S_{j}^{*}\right)=\sum_{j=1}^{n} a_{j} b_{j}=1,
$$

where we have used the relations (1.3).

Now take $\eta \in \Lambda[s, X]$ with the property $\delta_{b} \eta=0$. Then we get from (2.1) that $\delta_{b} \delta_{a^{*}}^{*} \eta=\eta$; hence by Proposition 2.2 the system $b$ is nonsingular.

Proposition 2.6. Suppose that the algebra $B$ is commutative. Then a system $b=\left(b_{1}, \ldots, b_{n}\right) \subset B$ is nonsingular if and only if there is a system $a=\left(a_{1}, \ldots, a_{n}\right)$ in $B$ such that

$$
a_{1} b_{1}+\ldots+a_{n} b_{n}=1 \text {. }
$$
2.5.

Proof. If such a system $a=\left(a_{1}, \ldots, a_{n}\right)$ exists, then $b$ is nonsingular by Lemma

Conversely, suppose that $b$ is nonsingular. Then the operator

$$
R(b)^{2}=\delta_{b} \delta_{b}^{*}+\delta_{b}^{*} \delta_{b}=\sum_{j=1}^{n} b_{j} b_{j}^{*}
$$

is invertible, where the calculation is similar with (2.1). If we define

$$
a_{j}=b_{j}^{*}\left(\sum_{j=1}^{n} b_{j} b_{j}^{*}\right)^{-1} \quad(j=1, \ldots, n),
$$

then the system $a=\left(a_{1}, \ldots, a_{n}\right)$ has the required property.

Proposition 2.6 shows that Definition 1.3 coincides with the classical definition of the joint spectrum in the commutative case (see, for instance [2]).

Another consequence of Lemma 2.5 is the following

COROLLARY 2.7. If $b=\left(b_{1}, \ldots, b_{n}\right)$ is a commuting system, then

$$
\sigma_{B}(b) \subset \sigma_{B}\left(b_{1}\right) \times \ldots \times \sigma_{B}\left(b_{n}\right) .
$$

LEMMA 2.8. Assume that $b^{\prime}=\left(b_{1}, \ldots, b_{n}, b_{n+1}, \ldots, b_{m}\right)$ is a commuting system in $B$. If $b=\left(b_{1}, \ldots, b_{n}\right)$ is nonsingular, then $b^{\prime}$ is nonsingular.

Proof. We may suppose that $m=1$ and an induction argument can be easily applied in order to yield the general result. As in the proof of Lemma 1.1, if $s^{\prime}$ $=\left(s_{1}, \ldots, s_{n}, s_{n+1}\right)$ then

$$
\Lambda\left[s^{\prime}, X\right]=\Lambda[s, X] \oplus S_{n+1} \Lambda[s, X] .
$$


Take $\eta \in \Lambda\left[s^{\prime}, X\right]$ such that $\delta_{b^{\prime}} \eta=0$. If $\eta^{\prime}+S_{n+1} \eta^{\prime \prime}$ is the canonical decomposition of $\eta$, we must have $\delta_{b} \eta^{\prime}=0$ and $b_{n+1} \eta^{\prime}=\delta_{b} \eta^{\prime \prime}$. Since $b$ is nonsingular, we can write $\eta^{\prime}=\delta_{b} \xi^{\prime}$, where $\xi^{\prime} \in \Lambda[s, X]$. Then $\delta_{b}\left(b_{n+1} \xi^{\prime}-\eta^{\prime \prime}\right)=0$, therefore $b_{n+1} \xi^{\prime}-$ $-\eta^{\prime \prime}=\delta_{b} \xi^{\prime \prime}$, with $\xi^{\prime \prime} \in \Lambda[s, X]$. It is easily seen that the form $\xi=\xi^{\prime}+S_{n+1} \xi^{\prime \prime}$ is a solution of the equation $\delta_{b^{\prime}}, \xi=\eta$; hence $b^{\prime}$ is nonsingular, by Proposition 2.2.

CoRollary 2.9. If $b=\left(b_{1}, \ldots, b_{n}\right)$ and $c=\left(c_{1}, \ldots, c_{m}\right)$ are systems in $B$ such that $(b, c)$ is commuting, then

$$
\sigma_{B}((b, c)) \subset \sigma_{B}(b) \times \sigma_{B}(c),
$$

where $(b, c)=\left(b_{1}, \ldots, b_{n}, c_{1}, \ldots, c_{m}\right)$.

THEOREM 2.10. If $b=\left(b_{1}, \ldots, b_{n}\right)$ and $c=\left(c_{1}, \ldots, c_{m}\right)$ are systems in $B$ such that $(b, c)$ is commuting, then

$$
\pi \sigma_{B}((b, c))=\sigma_{B}(b)
$$

where $\pi$ is the projection of $C^{n+m}$ onto the first $n$ coordinates.

Proof. The inclusion $\pi \sigma_{B}((b, c)) \subset \sigma_{B}(b)$ follows from Corollary 2.9. The other inclusion will be obtained from some technical results.

LemMA 2.11. Assume that $a=\left(a_{1}, \ldots, a_{n}\right) \subset \mathscr{L}(X)$ is a commuting system with $\mathscr{R}\left(\delta_{a}\right)$ not closed in $\Lambda[s, X]$. If $b \in \mathscr{L}(X)$ is in the commutant of $a, p$ is the projection of $A[s, X]$ onto $\mathscr{K}\left(\delta_{a}\right)$ and

$$
c_{w}^{*}=p(b-w)^{*} p+\delta_{a}^{*} p \quad(w \in \boldsymbol{C}),
$$

then there is a $w_{0} \in C$ such that the equation $d_{w_{0}} c_{w_{0}}^{*}=p$ has no solutions in $\mathscr{L}(\Lambda[s, X])$.

Proof of Lemma 2.11. Some arguments are inspired from [3]. Since $\mathscr{R}\left(\delta_{a}\right)$ is not closed, $\mathscr{R}\left(\delta_{a}^{*}\right)$ is not closed, either. Then we can find a sequence $\xi_{k} \in \overline{\mathscr{R}\left(\delta_{a}\right)}$ such that $\left\|\xi_{k}\right\|=1$ and $\delta_{a}^{*} \xi_{k} \rightarrow 0$ as $k \rightarrow \infty$. Let us define the vector states

$$
\varrho_{k}(\mu)=\left\langle\mu \xi_{k}, \xi_{k}\right\rangle \quad(\mu \in \mathscr{L}(\Lambda[s, X]) ; k=1,2,3, \ldots) .
$$

By passing to a subsequence, we may suppose that there is a state $\varrho$ on $\mathscr{L}(\Lambda[s, X])$ such that

$$
\varrho(\mu)=\lim _{k \rightarrow \infty}\left\langle\mu \xi_{k}, \xi_{k}\right\rangle \quad(\mu \in \mathscr{L}(\Lambda[s, X])) .
$$

Let $\varphi_{\mathrm{e}}: \mathscr{L}(\Lambda[s, X]) \rightarrow \mathscr{L}\left(X_{\mathrm{e}}\right)$ be the *-representation associated with $\varrho$, with the cyclic vector $\xi_{e}$ [4]. If $Y_{Q}$ is the kernel of $\varphi_{e}\left(\delta_{a}^{*}\right)$, then $Y_{e} \ni \xi_{e}$; hence $Y_{e} \neq\{0\}$. We have more, namely, $Z_{e}=\varphi_{e}(p) Y_{e}$ is not null. Indeed, $\varphi_{e}(p) \xi_{e} \ni Z_{e}$ and

$$
\left\|\varphi_{e}(p) \xi_{e}\right\|^{2}=\varrho(p)=\lim _{k \rightarrow \infty}\left\langle p \xi_{k}, \xi_{k}\right\rangle=1 \text {. }
$$

Since $\delta_{a}^{*} p=\delta_{a}^{*}$, we have $Z_{\varrho} \subset Y_{e}$. Consider now the operator $\varphi_{e}\left(p b^{*}\right) \mid Z_{e}$. As $\delta_{a}^{*} b^{*}=b^{*} \delta_{a}^{*}$, the values of $\varphi_{e}\left(p b^{*}\right) \mid Z_{e}$ are in $Z_{e}$. Take $\bar{w}_{0}$ in the boundary of the spectrum of $\varphi_{e}\left(p b^{*}\right) \mid Z_{\rho}$. Then we can find a sequence $\eta_{e}^{k} \in Z_{Q}$ with $\left\|\eta_{e}^{k}\right\|=1$ and $\left(\varphi_{e}\left(p b^{*}\right)-\bar{w}_{0}\right) \eta_{e}^{k}$ convergent to $0(k \rightarrow \infty)$. Suppose that the equation $d_{w_{0}} c_{w_{0}}^{*}=p$ has a bounded solution. Then

and letting $k \rightarrow \infty$ we obtain a contradiction.
LeMMA 2.12. Assume that $\alpha(w)=\left(a_{1}, \ldots, a_{n}, b-w\right)$ is a commuting system in $\mathscr{L}(X)$, where $w \in C$ is arbitrary. If $\alpha(w)$ is nonsingular for any $w \in C$, then $a=\left(a_{1}, \ldots\right.$ $\left.\ldots, a_{n}\right)$ is nonsingular.

Proof of Lemma 2.12. If $\alpha(w)$ is nonsingular, then we have in $A[s, X]$ the equality

$$
\mathscr{K}\left(\delta_{a}\right)=\mathscr{R}\left(\delta_{a}\right)+(b-w) \mathscr{K}\left(\delta_{a}\right) .
$$

- Indeed, if $\eta \in \mathscr{K}\left(\delta_{a}\right)$ then $\delta_{\alpha(w)} S_{n+1} \eta=0$, hence by the nonsingularity of $\alpha(w)$,

$$
S_{n+1} \eta=\delta_{a} \xi_{1}+S_{n+1}\left((b-w) \xi_{1}+\delta_{a} \xi_{2}\right),
$$

whence $\delta_{a} \xi_{1}=0$ and $\eta=(b-w) \xi_{1}+\delta_{a} \xi_{2}$, i.e.,

$$
\mathscr{K}\left(\delta_{a}\right) \subset \mathscr{R}\left(\delta_{a}\right)+(b-w) \mathscr{K}\left(\delta_{a}\right) .
$$

Since the opposite inclusion is obvious, we have (2.2).

If $\overline{\mathscr{R}\left(\delta_{a}\right)} \neq \mathscr{K}\left(\delta_{a}\right)$ and $q$ is the projection of $\mathscr{K}\left(\delta_{a}\right)$ onto $\mathscr{K}\left(\delta_{a}\right) \ominus \mathscr{R}\left(\delta_{a}\right)$, we infer from (2.2)

$$
\mathscr{K}\left(\delta_{a}\right) \ominus \mathscr{R}\left(\delta_{a}\right)=(q b-w) \mathscr{K}\left(\delta_{a}\right) \ominus \mathscr{R}\left(\delta_{a}\right)
$$

and this equality is not possible for any $w \in C$.

We may suppose then $\mathscr{R}\left(\delta_{a}\right) \neq \overline{\mathscr{R}\left(\delta_{a}\right)}=\mathscr{K}\left(\delta_{a}\right)$. Consider the operator

$$
c_{w}=\delta_{a}(1-p)+(b-w) p ;
$$

the notation being preserved from the previous lemma. If the equality (2.2) were true for any $w \in C$, then the equation $c_{w} d_{w}^{*}=p$ would have a bounded solution $d_{w}$ for any $w \in C$, which contradicts Lemma 2.11 .

Let us return to the proof of Theorem 2.10. It is enough to check the assertion for $m=1$. Take $z \in \sigma_{B}(b)$. According to the preceding lemma, we can find $w \in C$ such that $\left(b-z, c_{1}-w\right)$ is not singular, hence $(z, w) \in \sigma_{B}((b, c))$.

The property given by Theorem 2.10 is known as the projection property of the joint spectrum. An important consequence of it is the following

COROLLARY 2.13. For any commuting system $b=\left(b_{1}, \ldots, b_{n}\right)$ in $B$ the joint spectrum is nonempty.

Proof. By Theorem 2.10, the projection of $\sigma_{B}(b)$ onto the $j$ th coordinate is equal to $\sigma_{B}\left(b_{j}\right)(j=1, \ldots, n)$, which is a nonempty set.

Proposition 2.14. The mapping

$$
C^{n} \ni z \rightarrow R(z)=\delta_{z}+\delta_{x}^{*} \in M[s]
$$

is an R-linear isometry. Moreover, for any $z \neq 0$ we have $R(z)^{-1}=\|z\|^{-2} R(z)$, where $\left.\|z\|\right|^{2}=\left|z_{1}\right|^{2}+\ldots+\left|z_{n}\right|^{2}$.

Proof. A calculation similar to (2.1) shows that $R(z)^{2}=\delta_{z} \delta_{z}^{*}+\delta_{z}^{*} \delta_{z}=\|z\|^{2}$, whence $R(z)^{-1}=\|z\|^{-2} R(z)$. Since $R(z)$ is self-adjoint, we can write hence $z \rightarrow R(z)$ is an $R$-linear isometry. 
LEMMA 2.15. Consider a commuting system $b=\left(b_{1}, \ldots, b_{n}\right) \subset B$. If $z^{0} \notin \sigma_{B}(b)$ and $z \in C^{n}$ is such that $\left\|z-z^{0}\right\|<\left\|R\left(z^{0}-b\right)^{-1}\right\|^{-1}$, then $z \notin \sigma_{B}(b)$.

Proof. Indeed, by the previous proposition,

$$
\left\|R\left(z-z^{0}\right) R\left(z^{0}-b\right)^{-1}\right\|<1 ;
$$

therefore the series

$$
R\left(z^{0}-b\right)^{-1} \sum_{k=0}^{\infty}(-1)^{k}\left(R\left(z-z^{0}\right) R\left(z^{0}-b\right)^{-1}\right)^{k}
$$

is absolutely convergent in $M[s, B]$ and defines an inverse for $R(z-b)$ [5].

THEOREM 2.16. For any commuting system $b=\left(b_{1}, \ldots, b_{n}\right)$ in $B$ the set $\sigma_{B}(b)$ is a compact nonempty subset of $\boldsymbol{C}^{\boldsymbol{n}}$.

Proof. According to Corollary 2.13 the set $\sigma_{B}(b)$ is nonempty. From Corollary 2.7 we obtain that $\sigma_{B}(b)$ is bounded, while from Lemma 2.15 we derive that $\sigma_{B}(b)$ is closed.

We end this section with a result concerning a concept which relates the Gelfand theory of commutative Banach algebras and the notion of the joint spectrum, essentially due to Taylor [9].

Assume that $A \subset B$ is a commutative Banach algebra, whose identity coincides with that of $B$. We denote by $\Gamma(A)$ the Gelfand space of $A$. Let us define the set

$$
\Gamma(A ; B)=\bigcap_{b \subset A} \hat{b}^{-1}\left(\sigma_{B}(b)\right) \subset \Gamma(A),
$$

where $\hat{b}=\left(\hat{b}_{1}, \ldots, \hat{b}_{n}\right)$ is the Gelfand transform of the system $b=\left(b_{1}, \ldots, b_{n}\right)$ and $b$ is arbitrary in $A$.

Proposition 2.17. The set $\Gamma(A ; B)$ is nonempty. Moreover, $\hat{b}(\Gamma(A ; B))=\sigma_{B}(b)$ for any $b \subset A$.

Proof. Let us define $\Gamma_{b}=\hat{b}^{-1}\left(\sigma_{B}(b)\right)$. Notice that the sets $\Gamma_{b}$ are compact and nested downwards under inclusion. Indeed, the compactness is obvious; if $b^{1}, \ldots, b^{m}$ are finite systems in $A$ and $b=\left(b^{1}, \ldots, b^{m}\right)$ then $\Gamma_{b} \subset \Gamma_{b^{1}} \cap \ldots \cap \Gamma_{b^{m}}$, by Theorem 2.10. In this way, the intersection $\Gamma(A ; B)=\bigcap_{b \subset A} \Gamma_{b}$ is nonempty. Let us show that this set has the desired property. The inclusion $\hat{b}(\Gamma(A ; B)) \subset \sigma_{B}(b)$ is obvious. Conversely, take $z \in \sigma_{B}(b)$. If $\hat{b}^{-1}(z) \cap \Gamma(A ; B)=\varnothing$, we can choose $c \subset A$ such that $\hat{b}^{-1}(z) \cap \Gamma_{c}=\varnothing$. If $d=(b, c)$, take $w \in \sigma_{B}(c)$ with $(z, w) \in \sigma_{B}(d)$ hence $(z, w)=\hat{d}\left(\gamma_{1}\right)$ with $\gamma_{1} \in \Gamma_{d} \subset \Gamma_{c}$. Then $z=\hat{b}\left(\gamma_{1}\right)$, which is a contradiction.

\section{A generalized Martinelli kernel}

An important integral formula in the theory of analytic complex-valued functions in several variables is Martinelli's formula [6], [7], known also as the CauchyMartinelli-Bochner formula. Namely, if $U$ is an arbitrary open set in $C^{n}$ and $\Delta \subset U$ is a relatively compact open set whose boundary $\Sigma$ is piecewise smooth and is con- tained in $U$, then for every $f$ analytic in $U$ and any $w=\left(w_{1}, \ldots, w_{n}\right) \in \Delta$ we have the representation

$$
f(w)=\frac{(n-1) !}{(2 \pi i)^{n}} \int_{\Sigma} f(z) \sum_{j=1}^{n}(-1)^{j-1} \frac{\bar{z}_{j}-\bar{w}_{j}}{\|z-w\|^{2 n}} \omega_{j}(z),
$$

where

$$
\omega_{j}(z)=\bigwedge_{\substack{1 \leqslant k \leqslant n \\ k \neq j}} d \bar{z}_{k} \wedge d z_{1} \wedge \ldots \wedge d z_{n} .
$$

For $n=1$ the formula (3.1) reduces to the usual Cauchy formula. The differential form

$$
(n-1) ! \sum_{j=1}^{n}(-1)^{j-1} \frac{\bar{z}_{j}}{\|z\|^{2 n}} \bigwedge_{\substack{1 \leqslant k \leqslant n \\ k \neq j}} d \bar{z}_{k}
$$

will be called the Martinelli kernel. In this section we shall be looking for an analogue of the Martinelli kernel in $C^{*}$-algebras.

If $U \subset C^{n}$ is an open set and $Y$ is an arbitrary Banach space, we denote by $C^{\infty}(U, Y)$ [by $A(U, Y)$ ] the set of all $Y$-valued indefinitely differentiable [analytic] functions in $U$. When $Y=C$, then $C^{\infty}(U, Y)=C_{1}^{\infty}(U)[A(U, Y)=A(U)]$. If $z$ $=\left(z_{1}, \ldots, z_{n}\right)$ is the variable of the space, we denote by $d \bar{z}$ the system of indeterminates $\left(d \bar{z}_{1}, \ldots, d \bar{z}_{n}\right)$ and by $\bar{\partial}_{z}$ the differential operator

$$
\frac{\partial}{\partial \bar{z}_{1}} d \bar{z}_{1}+\ldots+\frac{\partial}{\partial \bar{z}_{n}} d \bar{z}_{n} .
$$

When $U \subset C^{n+m}$, we write the points of $U$ in the form $(z, w)=\left(z_{1}, \ldots, z_{n}, w_{1}, \ldots\right.$ $\left.\ldots, w_{m}\right)$. The corresponding operator $\bar{\partial}_{(z, w)}$ is then given by $\bar{\partial}_{z}+\bar{\partial}_{w}$. The operator $\bar{\partial}_{z}+\bar{\partial}_{w}$ will be also denoted by $\bar{\partial}$.

Consider again a fixed $C^{*}$-algebra with identity $B \subset \mathscr{L}(X)$. We work in this section mainly with commuting systems $\beta=\left(\beta_{1}, \ldots, \beta_{n}\right)$ in $A(U, B)$, i.e. systems for which $\beta_{j}(z) \beta_{k}(w)=\beta_{k}(w) \beta_{j}(z)$ for any $z, w \in U$ and $j, k=1, \ldots, n$.

Denote by $\sigma_{B}^{U}(\beta)$ the set of all $z \in U$ such that the system $\beta(z)=\left(\beta_{1}(z), \ldots\right.$ $\left.\ldots, \beta_{n}(z)\right)$ is singular in $U$. In this definition $U$ may be an open set in $C^{n+m}$. An argument similar to that used in the proof of Lemma 2.15 shows that the set $\sigma_{B}^{U}(\beta)$ is closed in $U$. Furthermore, the map

$$
R(\beta(z))^{-1}=\left(\delta_{\beta(z)}+\delta_{\beta(z)}^{*}\right)^{-1} \quad\left(z \in U \backslash \sigma_{B}^{U}(\beta)\right),
$$

having values in $M[s, B]$, is indefinitely differentiable (as a matter of fact, this map is actually $\boldsymbol{R}$-analytic, as one can easily see from a slightly modified proof of Lemma 2.15).

As we have mentioned, the notation $\Lambda[s, Y]\left[\Lambda^{p}[s, Y]\right]$ will always mean the space $Y \otimes \Lambda[s]\left[Y \otimes \Lambda^{p}[s]\right]$, for any linear space $Y$. In particular, we may consider the space $A\left((s, d \bar{z}, d \bar{w}), C^{\infty}(U, X)\right)$, where $U \subset C^{n+m}$ is open. If $\beta=\left(\beta_{1}, \ldots, \beta_{n}\right)$ $\subset A(U, B)$ is a commuting system, then the operator $\delta_{\beta}$ is defined on $A[(s, d \bar{z}, d \bar{w})$, 
$\left.C^{\infty}(U, X)\right]$ by the relation $\left(\delta_{\beta} \xi\right)(z, w)=\delta_{\beta(z, w)} \xi(z, w)$, for any $(z, w) \in U$. (For every pair of systems of linear transformations $b=\left(b_{1}, \ldots, b_{n}\right)$ and $c=\left(c_{1}, \ldots, c_{m}\right)$ we keep the notation $(b, c)$ for the system $\left(b_{1}, \ldots, b_{n}, c_{1}, \ldots, c_{m}\right)$. A similar notation will be used for systems of indeterminates.) We shall occasionally need a system of indeterminates $t=\left(t_{1}, \ldots, t_{m}\right)$ and the corresponding system of operators given by (1.1) will be then $\left(T_{1}, \ldots, T_{m}\right)$.

THEOREM 3.1. Let $U$ be an open set in $C^{n+m}$ and $\beta=\left(\beta_{1}, \ldots, \beta_{n}\right)$ a commuting system in $A(U, B)$, where $B \subset \mathscr{L}(X)$. Assume that

$$
\eta \in \Lambda\left[(s, d \bar{z}, d \bar{w}), C^{\infty}(U, \Lambda[t, X])\right]
$$

has the property $\left(\delta_{\beta}+\bar{\partial}\right) \eta=0$ in $U$. Then the equation $\left(\delta_{\beta}+\bar{\partial}\right) \xi=\eta$ has a solution

$$
\xi \in \Lambda\left[(s, d \bar{z}, d \bar{w}), C^{\infty}(V, \Lambda[t, X])\right],
$$

where $V \subset U \backslash \sigma_{B}^{U}(\beta)$ is an arbitrary open set.

Proof. With no loss of generality we may suppose that $\eta$ is homogeneous of degree $p \leqslant 2 n+m$ in $s_{1}, \ldots, s_{n}, d \bar{z}_{1}, \ldots, d \bar{z}_{n}, d \bar{w}_{1}, \ldots, d \bar{w}_{m}$. Let us represent $\eta$ $=\eta_{0}+\eta_{1}+\ldots+\eta_{p}$, where $\eta_{j}$ is of degree $j$ in $d \bar{z}_{1}, \ldots, d \bar{z}_{n}, d \bar{w}_{1}, \ldots, d \bar{w}_{m}$ and of degree $p-j$ in $s_{1}, \ldots, s_{n}$. We shall be looking for a solution of the form $\xi=\xi_{0}+$ $+\xi_{1}+\ldots+\xi_{p-1}$, where $\xi_{j}$ is of degree $j$ in $d \bar{z}_{1}, \ldots, d \bar{z}_{n}, d \bar{w}_{1}, \ldots, d \bar{w}_{m}$ and of degree $p-j-1$ in $s_{1}, \ldots, s_{n}$. Then the equation $\left(\delta_{\beta}+\bar{\partial}\right) \xi=\eta$ with the condition $\left(\delta_{\beta}+\bar{\partial}\right) \eta$ $=0$ is equivalent to the following system of equations with supplementary conditions:

$$
\begin{array}{l|l}
\delta_{\beta} \xi_{0}=\eta_{0} & \delta_{\beta} \eta_{0}=0 \\
\delta_{\beta} \xi_{1}+\bar{\partial} \xi_{0}=\eta_{1} & \delta_{\beta} \eta_{1}+\bar{\partial} \eta_{0}=0 \\
\cdots \cdots \cdots \cdots \cdots \cdots \cdots & \cdots \cdots \cdots \cdots \cdots \cdots \\
\delta_{\beta} \xi_{p-1}+\overline{\partial \xi_{p-2}}=\eta_{p-1} & \delta_{\beta} \eta_{p}+\bar{\partial} \eta_{p-1}=0 \\
\bar{\partial} \xi_{p-1}=\eta_{p} & \bar{\partial} \eta_{p}=0 .
\end{array}
$$

Let us find a solution of this system. We note that $\eta_{0}(z, w) \in \mathscr{K}\left(\delta_{\beta(z, w)}\right)$, for any $(z, w) \in U$. Define

$$
\xi_{0}(z, w)=R(\beta(z, w))^{-1} \eta_{0}(z, w) .
$$

From the proof of Lemma 2.3 we obtain $\xi_{0}(z, w) \in \mathscr{K}\left(\delta_{\beta(z, w)}^{*}\right)$ for any $(z, w)$ $\in V \subset U \backslash \sigma_{B}^{U}(\beta)$ and $\delta_{\beta} \xi_{0}=\eta_{0}$ in $V$. Moreover,

$$
\delta_{\beta} \bar{\partial} \xi_{0}=-\bar{\partial} \delta_{\beta} \xi_{0}=-\bar{\partial} \eta_{0}=\delta_{\beta} \eta_{1},
$$

whence $\delta_{\beta}\left(\eta_{1}-\bar{\partial} \xi_{0}\right)=0$. Define now

$$
\xi_{1}(z, w)=R(\beta(z, w))^{-1}\left(\eta_{1}(z, w)-\bar{\partial} \xi_{0}(z, w)\right) .
$$

We have as above $\xi_{1}(z, w) \in \mathscr{K}\left(\delta_{\beta(z, w)}^{*}\right)$ for any $(z, w) \in V$ and $\delta_{\beta} \xi_{1}=\eta_{1}-\bar{\partial} \xi_{0}$. Furthermore,

$$
\delta_{\beta}\left(\eta_{2}-\bar{\partial} \xi_{1}\right)=\delta_{\beta} \eta_{2}+\bar{\partial} \delta_{\beta} \xi_{1}=\delta_{\beta} \eta_{2}+\partial\left(\eta_{1}-\partial \xi_{0}\right)=0
$$

and the procedure can be continued. We obtain, in general,

$$
\eta_{j}(z, w)-\bar{\partial} \xi_{j-1}(z, w) \in \mathscr{K}\left(\delta_{\beta(z, w)}\right) \quad(j=1, \ldots, p) ;
$$

therefore

$$
\xi_{j}(z, w)=R(\beta(z, w))^{-1}\left(\eta_{j}(z, w)-\overline{\partial \xi_{j-1}}(z, w)\right) \in \mathscr{K}\left(\delta_{\beta(z, w)}^{*}\right)
$$

and

$$
\delta_{\beta} \xi_{J}+\overline{\partial \xi}_{j-1}=\eta_{J} \quad(j=1, \ldots, p-1),
$$

for any $(z, w) \in V$. We must now show that $\overline{\partial \xi}_{p-1}=\eta_{p}$. From the proof of Lemma 2.3 and from the structure of the operator defined by (1.5), it follows easily that $R(\beta(z, w))^{-1}$ maps the kernel of $\delta_{\beta(z, w)}$ in $\Lambda^{q+1}[s, \Lambda[t, X]]$ into the kernel of $\delta_{\beta(z, w)}^{*}$ in $\Lambda^{q}[s, \Lambda[t, X]]$ for every $q$; therefore the degree of $\xi_{j}(z, w)$ in $s_{1}, \ldots, s_{n}$ must be $p-j-1$. In this way. we obtain that the degree of $\eta_{p}(z, w)-\bar{\partial} \xi_{p-1}(z, w)$ in $s_{1}, \ldots, s_{n}$ is zero. Since the kernel of $\delta_{\beta(z, w)}$ in $\Lambda^{0}[s, A[t, X]]$ is zero for each $(z, w) \in V$, we obtain $\eta_{p}=\bar{\partial} \bar{\xi}_{p-1}$, and this finishes the proof.

Remark 3.2. The formula (3.3) can be written explicitly as

$$
\xi_{j}(z, w)=\sum_{k=0}^{j}(-1)^{k} R(\beta(z, w))^{-1}\left(\bar{\partial} R(\beta(z, w))^{-1}\right)^{k} \eta_{j-k}(z, w),
$$

for every $j=0,1, \ldots, p-1$. In other words, from Theorem 3.1 we have obtained the following result: If we take

$$
\eta \in \Lambda^{p}\left[(s, d \bar{z}, d \bar{w}), C^{\infty}(U, \Lambda[t, X])\right]
$$

which satisfies $\left(\delta_{\beta}+\bar{\partial}\right) \eta=0$ in $U$, then the form

$$
H(\beta) \eta(z, w)=\sum_{j=0}^{p-1} \sum_{k=0}^{j}(-1)^{k} R(\beta(z, w))^{-1}\left(\bar{\partial} R(\beta(z, w))^{-1}\right)^{k} \eta_{j-k}(z, w)
$$

is a solution of the equation $\left(\delta_{\beta}+\bar{\partial}\right) \xi=\eta$ in the space

$$
\Lambda^{p-1}\left[(s, d \bar{z}, d \bar{w}), C^{\infty}(V, \Lambda[t, X])\right],
$$

where $V \subset U \backslash \sigma_{B}^{U}(\beta)$ is an open set and $\eta_{j}$ is the part of $\eta$ of degree $j$ in $d \bar{z}_{1}, \ldots, d \bar{z}_{n}$, $d \bar{w}_{1}, \ldots, d \bar{w}_{m}$. In a certain sense, $H(\beta) \eta$ is the canonical solution of the equation $\left(\delta_{\beta}+\bar{\partial}\right) \xi=\eta$.

We shall use in what follows the notation

$$
S=S_{1} \ldots S_{n} .
$$

Analogously, we denote by $T$ the operator $T_{1} \ldots T_{m}$.

Proposition. 3.3. Consider a commuting system $\beta=\left(\beta_{1}, \ldots, \beta_{n}\right)$ in $A(U, B)$,

31 Banach Center t. VIII 
where $U \subset C^{n}$ is an open set. If $V \subset U \backslash \sigma_{B}^{U}(\beta)$ is an arbitrary open set, then

$$
M(\beta(z))=R(\beta(z))^{-1}\left(\bar{\partial}_{z} R(\beta(z))^{-1}\right)^{n-1} S \mid X \quad(z \in V)
$$

is a differential form in $\Lambda^{n-1}\left[d \bar{z}, C^{\infty}(V, B)\right]$ with the property $\bar{\partial}_{z} M(\beta(z))=0$ in $V$.

Proof. We apply Theorem 3.1 with $m=0$. If we fix $x \in X$ and define $\eta(z)$ $=\eta_{0}(z)=S x$, then the coefficients of $\xi_{0}(z)$ (see the proof of Theorem 3.1) are given by continuous operators acting from $X$ into $\Lambda^{n-1}[s, X]$, calculated in $x$. We shall obtain by induction that

$$
\xi_{j}(z)=(-1)^{j} R(\beta(z))^{-1}\left(\bar{\partial}_{z} R(\beta(z))^{-1}\right)^{j} S x
$$

has coefficients given by continuous operators from $X$ into $\Lambda^{n-j-1}[s, X]$, calculated in $x$, for any $j=0,1, \ldots, n-1$. Notice that $M(\beta(z)) x=(-1)^{n-1} \xi_{n-1}(z)$; since $\eta_{n}(z)=0$, we must have $\bar{\partial}_{z} M(\beta(z))=0$ in $V$ and the coefficients of $M(\beta(z))$ are operators from $\mathscr{L}(X)$. Finally, from the uniqueness of the representation of the elements of $M[s, B]$ (see Lemma 1.1), we obtain that the coefficients of $M(\beta(z))$ are actually elements of $B$, for any $z \in V$, and the proof is finished.

DefinItion 3.4. The differential form (3.5) will be called the Martinelli kernel attached to the commuting system $\beta=\left(\beta_{1}, \ldots, \beta_{n}\right) \subset A(U, B)$.

The following result will show that Definition 3.4 extends naturally the usual Martinelli kernel given by (3.2).

Theorem 3.5. Consider the scalar system $z=\left(z_{1}, \ldots, z_{n}\right) \subset A\left(C^{n}\right)$. Then for any $z \neq 0$ we have the equality

$$
M(z)=(n-1) ! \sum_{j=1}^{n}(-1)^{j-1} \frac{\bar{z}_{j}}{\|z\|^{2 n}} \bigwedge_{\substack{1<k<n \\ k \neq j}} d \bar{z}_{k},
$$

the coefficients of the right side being multiplication operators.

Proof. It will be enough to prove the equality

$$
\begin{aligned}
M(z)(1) & =R(z)^{-1}\left(\bar{\partial}_{z} R(z)^{-1}\right)^{n-1} s_{1} \wedge \ldots \wedge s_{n} \\
& =(n-1) ! \sum_{j=1}^{n}(-1)^{j-1} \frac{\bar{z}_{J}}{\|z\|^{2 n}} \bigwedge_{\substack{1<k<n \\
k \neq j}} d \bar{z}_{k} .
\end{aligned}
$$

We have $R(z)^{-1}=\|z\|^{-2}\left(\delta_{z}+\delta_{z}^{*}\right)$, by Proposition 2.14. From the proof of Theorem 3.1 we infer easily

(3.7) $R(z)^{-1}\left(\bar{\partial}_{z} R(z)^{-1}\right)^{n-1} s_{1} \wedge \ldots \wedge s_{n}=\frac{\delta_{z}^{*}}{\|z\|^{2}}\left(\bar{\partial}_{z} \frac{\delta_{z}^{*}}{\|z\|^{2}}\right)^{n-1} s_{1} \wedge \ldots \wedge s_{n}$.

Let us define the operators

$$
R_{f}(\xi)=\bar{\partial}_{z}\left(\bar{z}_{j}\|z\|^{-2}\right) \wedge \xi \quad(j=1, \ldots, n),
$$

and note that $R_{j} R_{k}=-R_{k} R_{j}$ and $R_{j} S_{k}^{*}=-S_{k}^{*} R_{j}$, for any pair of indices $j$ and $k$ Then we can write

$$
\begin{aligned}
\frac{\delta_{z}^{*}}{\|z\|^{2}}\left(\bar{\partial}_{z}\right. & \left.\frac{\delta_{z}^{*}}{\|z\|^{2}}\right)^{n-1} s_{1} \wedge \ldots \wedge s_{n} \\
& =\frac{\delta_{z}^{*}}{\|z\|^{2}}\left(\sum_{j_{1}, \ldots, j_{n-1}=1}^{n} R_{j_{1}} S_{j_{1}}^{*} \ldots R_{j_{n-1}} S_{j_{n-1}}^{*}\right) s_{1} \wedge \ldots \wedge s_{n} \\
& =c_{n} \frac{\delta_{z}^{*}}{\|z\|^{2}}\left(\sum_{j_{1}, \ldots, j_{n-1}=1}^{n} R_{j_{1}} \ldots R_{j_{n-1}} S_{j_{1}}^{*} \ldots S_{j_{n-1}}^{*}\right) s_{1} \wedge \ldots \wedge s_{n} \\
& =c_{n}(n-1) ! \frac{\delta_{z}^{*}}{\|z\|^{2}}\left(\sum_{j=1}^{n} R_{1} \ldots \hat{R}_{j} \ldots R_{n} S_{1}^{*} \ldots \widehat{S_{j}^{*}} \ldots S_{n}^{*}\right) s_{1} \wedge \ldots \wedge s_{n},
\end{aligned}
$$

where $c_{n}=(-1)^{(n-1)(n-2) / 2}$. Since

$$
s_{1} \wedge s_{2} \wedge \ldots \wedge s_{n}=(-1)^{n(n-1) / 2} s_{n} \wedge s_{n-1} \wedge \ldots \wedge s_{1},
$$

we obtain, on account of the previous equalities,

$$
\begin{aligned}
\frac{\delta_{z}^{*}}{\|z\|^{2}}\left(\bar{\partial}_{z} \frac{\delta_{z}^{*}}{\|z\|^{2}}\right)^{n-1} & s_{1} \wedge \ldots \wedge s_{n} \\
& =(n-1) ! \sum_{j=1}^{n} \frac{\bar{z}_{j} S_{j}^{*}}{\|z\|^{2}} \sum_{k=1}^{n}(-1)^{k-1} S_{k} \wedge R_{1} \ldots \hat{R}_{k} \ldots R_{n}(1) \\
& =(n-1) ! \sum_{j=1}^{n}(-1)^{j-1} \frac{\bar{z}_{j}}{\|z\|^{2}} \bigwedge_{\substack{1 \leq k<n \\
k \neq j}}\left(\bar{\partial}_{z} \frac{\bar{z}_{k}}{\|z\|^{2}}\right) .
\end{aligned}
$$

By using the relations

$$
\bar{\partial}_{z}\left(\bar{z}_{k}\|z\|^{-2}\right)=\bar{z}_{k} \bar{\partial}_{z}\|z\|^{-2}+\|z\|^{-2} d \bar{z}_{k} \quad(k=1, \ldots, n)
$$

and

$$
\left(\bar{\partial}_{z}\|z\|^{-2}\right) \wedge\left(\bar{\partial}_{z}|| z||^{-2}\right)=0,
$$

we can write for $n \geqslant 3$ (and similarly for $n=1,2$ )

$$
\begin{aligned}
& \sum_{j=1}^{n}(-1)^{j-1} \frac{\bar{z}_{j}}{\|z\|^{2}} \bigwedge_{k \neq j}\left(\bar{\partial}_{z} \frac{\bar{z}_{k}}{\|z\|^{2}}\right) \\
& =\sum_{j=1}^{n}(-1)^{j-1} \frac{\bar{z}_{j}}{\|z\|^{2 n-2}}\left(\sum_{p<j}(-1)^{p-1} \bar{z}_{p} \bar{\partial}_{z}\|z\|^{-2} \wedge \bigwedge_{\substack{q \neq p \\
q \neq j}} d \bar{z}_{q}+\right. \\
& \left.\quad+\sum_{p>j}(-1)^{p} \bar{z}_{p} \bar{\partial}_{z}\|z\|^{-2} \wedge \bigwedge_{\substack{q \neq p \\
q \neq j}} d \bar{z}_{q}\right)+\sum_{j=1}^{n}(-1)^{j-1} \frac{\bar{z}_{j}}{\|z\|^{2 n}} \bigwedge_{k \neq j} d \bar{z}_{k}
\end{aligned}
$$




$$
\begin{aligned}
= & \frac{1}{\|z\|^{2 n-2}} \sum_{p<j}(-1)^{j+p} \bar{z}_{j} \bar{z}_{p} \bar{\partial}_{z}\|z\|^{-2} \wedge \bigwedge_{\substack{q \neq p \\
q \neq j}} d \bar{z}_{q}+ \\
& +\frac{1}{\|z\|^{2 n-2}} \sum_{p>j}(-1)^{j+p-1} \bar{z}_{j} \bar{z}_{p} \bar{\partial}_{z}\|z\|^{-2} \wedge \bigwedge_{\substack{q \neq p \\
q \neq j}} d \bar{z}_{q}+ \\
& +\sum_{j=1}^{n}(-1)^{j-1} \frac{\bar{z}_{j}}{\|z\|^{2 n}} \bigwedge_{k \neq j} d \bar{z}_{k} \\
= & \sum_{j=1}^{n}(-1)^{j-1} \frac{\bar{z}_{j}}{\|z\|^{2 n}} \bigwedge_{k \neq j} d \bar{z}_{k},
\end{aligned}
$$

which shows that (3.7) holds. As (3.7) is equivalent to (3.6), which in turn is just our assertion, the proof is complete.

Let $K$ be a compact set in $C^{n}$ and $U$ an open neighbourhood of $K$. It is always possible to find a relatively compact open set $\Delta \supset K$, with $\bar{\Delta} \subset U$, such that the boundary $\Sigma=\partial \Delta$ is a smooth surface. In this case we say that $\Sigma$ is an admissible surface surrounding $K$ in $U$.

If $\eta \in \Lambda\left[(s, d \bar{z}), C^{\infty}(U, X)\right]$ is an arbitrary element, then we define, as usually,

$$
\int_{U} \eta(z) \wedge d z_{1} \wedge \ldots \wedge d z_{n}=\int_{U} \eta_{n}(z) \wedge d z_{1} \wedge \ldots \wedge d z_{n},
$$

provided that the coefficients of $\eta_{n}(z)$ are integrable functions, where $\eta_{n}(z)$ is the part of $\eta(z)$ of degree $n$ in $d \bar{z}_{1}, \ldots, d \bar{z}_{n}$. define

Analogously, if $\Sigma \subset U$ is a smooth surface of real dimension $2 n-1$, then we

$$
\int_{\Sigma} \eta(z) \wedge d z_{1} \wedge \ldots \wedge d z_{n}=\int_{\Sigma} \eta_{n-1}(z) \wedge d z_{1} \wedge \ldots \wedge d z_{n},
$$

where $\eta_{n-1}(z)$ is the part of $\eta(z)$ of degree $n-1$ in $d \bar{z}_{1}, \ldots, d \bar{z}_{n}$. These integrals are, in general, elements of $A[s, X]$. Note also that we may consider instead of the space $X$ an arbitrary Banach space, in particular the algebra $B$.

For the sake of simplicity, we denote by $d Z$ the form $d z_{1} \wedge \ldots \wedge d z_{n}$. Similarly, the form $d w_{1} \wedge \ldots \wedge d w_{m}$ will be denoted by $d W$. From now on we denote also by $P$ the projection of the space $\Lambda\left[(s, t, d \bar{z}, d w), C^{\infty}(U, X)\right]$ onto the space $\Lambda[(d \bar{z}, d \bar{w})$, $C^{\infty}(U, X)$ ], where $U \subset C^{n+m}$ is open.

A closed set $F \subset U$ is said to be $C^{n}$-compact if for every compact $K \subset V$, the set $F \cap\left(C^{n} \times K\right)$ is compact in $U$, where $V$ is the projection of $U$ onto the last $m$ coordinates.

For some technical reasons, we need an extension of Definition 3.5. Namely, if $\eta \in \Lambda\left[(d \bar{z}, d \bar{w}), C^{\infty}(U, X)\right]$ has the property $\overline{\partial \eta}=0$, we put

$$
M(\beta) \eta=(-1)^{n-1} P H(\beta) S \eta,
$$

where $\beta=\left(\beta_{1}, \ldots, \beta_{n}\right) \subset A(U, B)$ is a commuting system. From Theorem 3.1 we bave still $\bar{\partial} M(\beta) \eta=0$ in $U \backslash \sigma_{B}^{v}(\beta)$.
If $\sigma_{B}^{U}(\beta)$ is $C^{n}$-compact in $U$ and $\varphi \in C^{\infty}(U)$ is a function such that the support of $\psi=1-\varphi$ is $C^{n}$-compact and $\psi=1$ in a neighbourhood of $\sigma_{B}^{v}(\beta)$, then we define for any $f \in A(U, X)$

$$
v_{\beta}(f)=\frac{1}{(2 \pi i)^{n}} \int \bar{\partial} \varphi M(\beta) f \wedge d Z .
$$

In particular, when $U \subset C^{n}$ and $\sigma_{B}^{U}(\beta)$ is compaçt, then

$$
v_{\beta}(f)=\frac{1}{(2 \pi i)^{n}} \int_{\Sigma} M(\beta) f \wedge d Z,
$$

where $\Sigma$ is an admissible surface surrounding $\sigma_{B}^{U}(\beta)$ in $U$, by applying the Stokes formula. From the formula (3.10) we shall eventually derive the analytic functional calculus. The integral (3.9) does.not depend on the particular choice of the function $\varphi$. This fact will follow from a more general result.

Lemma 3.6. Assume that $\beta=\left(\beta_{1}, \ldots, \beta_{n}\right)$ is a commuting system in $A(U, B)$, $U \subset C^{n+m}$, such that $\sigma_{B}^{U}(\beta)$ is $C^{n}$-compact in $U$. Consider a form

$$
\eta \in \Lambda^{n+p}\left[(s, t, d \bar{z}, d \bar{w}), C^{\infty}(U, X)\right]
$$

such that $\left(\delta_{\beta}+\bar{\partial}\right) \eta=0$ in $U$. If the support of $\eta$ is $C^{n}$-compact and disjoint with $\sigma_{B}^{U}(\beta)$ then we have the relation

$$
\int P \eta \wedge d Z=\bar{\partial}_{w} \xi,
$$

where $\xi \in A^{p-1}\left[d \bar{w}, C^{\infty}(V, X)\right]$ and $V$ is the projection of $U$ onto the last $m$ coordinates. When either $p=0$ or $m=0$, then $\xi=0$.

Proof. Indeed, the canonical solution $H(\beta) \eta$ of the equation $\left(\delta_{\beta}+\bar{\partial}\right) \xi=\eta$ is everywhere defined in $U$, and so $P \eta=\bar{\partial} \xi_{1}$, where the support of $\xi_{1}$ is $C^{n}$-compact. We have $\xi_{1}=\xi_{1, n}+\xi_{1}^{\prime}$, where $\xi_{1, n}$ is the part of $\xi_{1}$ of degree $n$ in $d \bar{z}_{1}, \ldots, d \bar{z}_{n}$. Then we can write, by the Stokes formula,

$$
\int P \eta \wedge d Z=\int \bar{\partial} \xi_{1, n} \wedge d Z+\int \bar{\partial} \xi_{1}^{\prime} \wedge d Z=\bar{\partial}_{w} \int \xi_{1, n} \wedge d Z=\bar{\partial}_{w} \xi .
$$

If $p=0$ then $\xi_{1, n}=0$ and if $m=0$ then $\bar{\partial}_{z} \xi_{1}=\bar{\partial}_{z} \xi_{1}^{\prime}$; in either case we obtain $\xi=0$. form

Now, if $\varphi_{1}$ and $\varphi_{2}$ are two different functions in the formula (3.9), then the

$$
\eta=P\left(\delta_{\beta}+\bar{\partial}\right)\left(\varphi_{1} H(\beta) S f-\varphi_{2} H(\beta) S f\right) \in \Lambda^{n}\left[(s, d \bar{z}, d \bar{w}), C^{\infty}(U, X)\right]
$$

has the properties from Lemma 3.6, whence our assertion follows.

If $\beta=\left(\beta_{1}, \ldots, \beta_{n}\right) \subset A(U, B)$ is a commuting system, where $U \subset C^{n+m}$, then for any $w$ belonging to the projection of $U$ onto the last $m$ coordinates the system $\beta_{w}$, defined by $\beta_{w}(z)=\beta(z, w)$, is commuting in $A\left(U_{w}, B\right), U_{w}$ being the set $\{z$ $\left.\in C^{n} ;(z, w) \in U\right\}$. Similarly, if $f \in A(U, X)$. then $f_{w} \in A\left(U_{w}, X\right)$, where $f_{w}(z)$ $=f(z, w)$. 
LEMMA 3.7. Assume that $\beta=\left(\beta_{1}, \ldots, \beta_{n}\right) \subset A(U, B)$ is a commuting system, where $U \subset C^{n+m}$ is open and $\sigma_{B}^{U}(\beta)$ is $C^{n}$-compact. Then for every $f \in A(U, X)$ we have

$$
v_{\beta}(f)(w)=v_{\beta_{w}}\left(f_{w}\right),
$$

where $w$ belongs to the projection of $U$ onto the last $m$ coordinates. Moreover, $v_{\beta}(f)$ is analytic.

Proof. Let $Q$ be the projection of $A\left[(s, t, d \bar{z}, d \bar{w}), C^{\infty}(U, X)\right]$ onto the subspace $\Lambda\left[(s, d \bar{z}), C^{\infty}(U, X)\right]$ and let us define the map

Note the relation

$$
Q_{w} \xi=(Q \xi)(w) \in \Lambda\left[(s, d \bar{z}), C^{\infty}\left(U_{w}, X\right)\right] .
$$

$$
Q_{w}\left(\left(\delta_{\beta}+\bar{\partial}\right) \xi\right)=\left(\delta_{\beta_{w}}+\overline{\partial_{z}}\right) Q_{w}(\xi)
$$

for any $\xi \in \Lambda\left[(s, t, d \bar{z}, d w), C^{\infty}(U, X)\right]$. Then the form

$$
\left(\bar{\delta}_{\beta_{w}}+\bar{\partial}_{z}\right)\left(\varphi_{w} H\left(\beta_{w}\right) S f_{w}-Q_{w}(\varphi H(\beta) S f)\right)
$$

has its support compact and disjoint with

$$
\sigma_{B}^{U w}\left(\beta_{w}\right) \subset\left(C^{n} \times\{w\}\right) \cap \sigma_{B}^{v}(\beta) .
$$

By Lemma 3.6 we can write

$$
\int \bar{\partial}_{z} \varphi_{w} M\left(\beta_{w}\right) f_{w} \wedge d Z=\int \bar{\partial}_{z} Q_{w}(\varphi M(\beta) f) \wedge d Z=\left(\int \bar{\partial} \varphi M(\beta) f \wedge d Z\right)(w) .
$$

Notice also that the form $\left(\delta_{\beta}+\bar{\partial}\right) \bar{\partial}_{w} \varphi H(\beta) S f$ is null in a neighbourhood of $\sigma_{B}^{U}(\beta)$ and its support is $C^{n}$-compact in $U$; hence by Lemma 3.6

$$
\bar{\partial}_{w} \int \bar{\partial} \varphi M(\beta) f \wedge d Z=0,
$$

i.e., $v_{\beta}(f)$ is analytic.

The next result is a Fubini type theorem for the integral (3.9).

THEOREM 3.8. Let $U$ be an open set in $C^{n+m}$ and $V$ the projection of $U$ onto the last $m$ coordinates. Consider also $\beta=\left(\beta_{1}, \ldots, \beta_{n}\right) \subset A(U, B)$ and $\gamma=\left(\gamma_{1}, \ldots, \gamma_{m}\right)$ $\subset A(V, B)$ such that $(\beta, \gamma)$ is a commuting system. If $\sigma_{B}^{U}(\beta)$ is $C^{n}$-compact in $U$ and $\sigma_{B}^{\nabla}(\gamma)$ is compact in $V$, then for any $f \in A(U)$ and $g \in A(V, X)$ we have the equality

$$
\nu_{(\beta, y)}(f g)=\nu_{\gamma}\left(\nu_{\beta}(f) g\right) \text {. }
$$

Proof. Let $\varphi$ be in $C^{\infty}(U)$ with the properties from the definition (3.9) for $\sigma_{B}^{U}(\beta)$. Analogously, take $\psi \in C^{\infty}(V)$ and $\theta \in C^{\infty}(U)$ with similar properties, corresponding to $\sigma_{B}^{\nabla}(\gamma)$ and

$$
\sigma_{B}^{U}((\beta, \gamma)) \subset \sigma_{B}^{U}(\beta) \cap\left(C^{n} \times \sigma_{B}^{V}(\gamma)\right)
$$

respectively. Let us define $\xi(z, w)=S f(z, w) g(w)$ and notice that $\left(\delta_{\beta}+\bar{\partial}\right) \xi=0$ in $U$. Then the support of $\eta_{0}=\left(\delta_{\beta}+\bar{\partial}\right) \varphi H(\beta) \xi-\xi$ is $C^{n}$-compact. Consider the form whity

$$
\eta_{1}=\left(\delta_{\beta}+\delta_{\gamma}+\bar{\partial}\right)(-1)^{m} \varphi T H(\beta) \xi-T \xi=T \eta_{0}
$$

and define

$$
\eta_{2}=-\left(\delta_{\beta}+\delta_{\gamma}+\bar{\partial}\right) \psi H((\beta, \gamma)) \eta_{1}+\eta_{1}
$$

and

$$
\tilde{\eta}_{2}=\left(\delta_{\beta}+\delta_{\gamma}+\bar{\partial}\right) \theta H((\beta, \gamma)) T \xi-T \xi .
$$

Since the support of $\eta_{1}$ is $\boldsymbol{C}^{n}$-compact, it is easily seen that the support of $\eta_{2}$ is compact. Moreover, when $\varphi=0$ and $\psi=0$, then $\eta_{2}=-T \xi$, i.e., $\eta_{2}=-T \xi$ in a neighbourhood of $\sigma_{B}^{U}((\beta, \gamma))$. Clearly, the support of $\tilde{\eta}_{2}$ is compact and $\tilde{\eta}_{2}$ $=-T \xi$ in a neighbourhood of $\sigma_{B}^{U}((\beta, \gamma))$. By Theorem 3.1 we may represent the difference $\eta_{2}-\tilde{\eta}_{2}$ as

$$
\eta_{2}-\tilde{\eta}_{2}=\left(\delta_{\beta}+\delta_{\gamma}+\bar{\partial}\right) \xi_{1}
$$

where the support of $\xi_{1}$ is compact.

Let $P_{1}$ be the projection of $\Lambda\left[(s, t, d \bar{z}, d \bar{w}), C^{\infty}(U, X)\right]$ onto the subspace $\Lambda\left[(t, d \bar{z}, d \bar{w}), C^{\infty}(U, X)\right]$ and consider the forms

and

$$
\zeta_{1}=\left(\delta_{\gamma}+\bar{\partial}\right) \psi H(\gamma) T P_{1} \eta_{0}-T P_{1} \eta_{0}
$$

$$
\tilde{\zeta}_{1}=P_{1}\left(\left(\delta_{\beta}+\delta_{\gamma}+\bar{\partial}\right) \psi H((\beta, \gamma)) \eta_{1}-\eta_{1}\right)
$$

Note that both $\zeta_{1}$ and $\tilde{\zeta}_{1}$ have their supports compact and both are equal to $-T P_{1} \eta_{0}$ when $\psi=0$; therefore we may write

(3.12)

$$
\zeta_{1}-\tilde{\zeta}_{1}=\left(\delta_{\gamma}+\bar{\partial}\right) \xi_{2},
$$

where the support of $\xi_{2}$ is compact. We shall then have

$$
\zeta=P \zeta_{1}=\bar{\partial} \psi P H(\gamma) T \bar{\partial} \varphi P H(\beta) \xi=(-1)^{m+n} \bar{\partial} \psi M(\gamma) \bar{\partial} \varphi M(\beta) f g
$$
and, by (3.11),

$$
\begin{aligned}
\tilde{\zeta} & =P \tilde{\zeta}_{1}=P\left(\left(\delta_{\beta}+\delta_{\gamma}+\bar{\partial}\right) \psi H((\beta, \gamma)) \eta_{1}-\eta_{1}\right) \\
& =-P\left(\left(\delta_{\beta}+\delta_{\gamma}+\bar{\partial}\right) \theta H((\beta, \gamma)) T \xi-T \xi\right)+\overline{\partial \xi_{3}} \\
& =-(1)^{m+n} \bar{\partial} \theta M((\beta, \gamma)) f g+\overline{\partial \xi}_{3},
\end{aligned}
$$

where the support of $\xi_{3}$ is compact. From (3.12) and from the above calculations we get

(3.13)

$$
\bar{\partial} \theta M((\beta, \gamma)) f g-\bar{\partial} \psi M(\gamma) \bar{\partial} \varphi M(\beta) f g=\bar{\partial} \xi_{0},
$$

where the support of $\xi_{0}$ is compact.

Note that by the Stokes formula, for $w \notin \sigma_{B}^{Y}(\gamma)$,

$$
\int \bar{\partial}_{z} H(\gamma) T \bar{\partial} \varphi M(\beta) f g \wedge d Z=0 .
$$

It is also clear that

$$
\int \bar{\partial} \varphi M(\beta) f g \wedge d Z=\left(\int \bar{\partial} \varphi M(\beta) f \wedge d Z\right) g ;
$$


therefore the form

$$
\left(\delta_{\gamma}+\bar{\partial}_{w}\right)\left(\psi \int H(\gamma) T \bar{\partial} \varphi M(\beta) f g \wedge d Z-\psi H(\gamma) T\left(\int \bar{\partial} \varphi M(\beta) f \wedge d Z\right) g\right)
$$

is zero in a neighbourhood of $\sigma_{B}^{\gamma}(\gamma)$ and its support is compact. By Lemma 3.6 we obtain

$$
\begin{aligned}
\int \bar{\partial} \varphi M(\gamma) \bar{\partial} \varphi M(\beta) f g \wedge d Z \wedge d W & =\int(\bar{\partial} \bar{\partial} \psi M(\gamma) \bar{\partial} \varphi M(\beta) f g \wedge d Z) \wedge d W \\
& =\int \bar{\partial}_{w} \psi M(\gamma)\left(\int \bar{\partial} \varphi M(\beta) f \wedge d Z\right) g \wedge d W,
\end{aligned}
$$

whence by the equality (3.13) we infer our assertion.

Let us denote by $B_{\beta}$ the double commutant in $B$ of the set

$$
\left\{\beta_{1}(z, w), \ldots, \beta_{n}(z, w) ;(z, w) \in U\right\},
$$

where $\beta=\left(\beta_{1}, \ldots, \beta_{n}\right) \subset A(U, B)$ is a commuting system. Then $B_{\beta}$ is a commutative Banach algebra with identity.

Proposition 3.9. With the conditions of Lemma 3.7 we have $v_{\beta}(f) \in A\left(V, B_{\beta}\right)$, for any $f \in A(U)$, where $V$ is the projection of $U$ onto the last $m$ coordinates.

Proof. If $b \in B$ is an element commuting with $\beta_{j}(z, w)$ for any $j=1, \ldots, n$ and $(z, w) \in U$, then we can consider the form

$$
\left(\delta_{\beta}+\bar{\partial}\right)(\varphi H(\beta) f b x-b \varphi H(\beta) f x) \quad(x \in X),
$$

which is null in a neighbourhood of $\sigma_{B}^{U}(\beta)$; hence from Lemma 3.6 we obtain the conclusion.

Remark 3.10. Proposition 3.9 shows that we can obtain a variant of Theorem 3.8 with a similar statement, taking for $f$ and $g$ two analytic $B_{(\beta, \gamma)}$-valued functions.

LeMma 3.11. If $\beta \in A(U, B)$ and $\sigma_{B}^{U}(\beta)$ is compact in $U \subset C$, then $\beta(z)$ is invertible if $z \in U \backslash \sigma_{B}^{U}(\beta)$ and we have

$$
\boldsymbol{v}_{\beta}(f)=\frac{1}{2 \pi i} \int_{\boldsymbol{T}} \beta(z)^{-1} f(z) d z \quad(f \in A(U, X)),
$$

where $T$ is an admissible contour surrounding $\sigma_{B}^{U}(\beta)$ in $U$.

Proof. Plainly, outside the set $\sigma_{B}^{U}(\beta)$ the function $\beta(z)$ must be invertible. Then we have $R(\beta(z))^{-1}=\beta(z)^{-1} S_{1}^{*}+\beta(z)^{*-1} S_{1}$, for any $z \in U \backslash \sigma_{B}^{U}(\beta)$ (Proposition 1.4). From the formula (3.10) we obtain

$$
v_{\beta}(f)=\frac{1}{2 \pi i} \int_{\Gamma} R(\beta(z))^{-1} S_{1} f(z) d z=\frac{1}{2 \pi i} \int_{\Gamma} \beta(z)^{-1} f(z) d z .
$$

Propositron 3.12. Let $\beta=\left(\beta_{1}, \ldots, \beta_{n}\right)$ be a commuting system in $A(U, B)$, where $U=U_{1} \times U_{2} \times \ldots \times U_{n}$. If $U_{j} \supset \sigma_{B}^{U}\left(\beta_{j}\right)$, which are compact, and, for each $j, \Gamma_{j}$ is an admissible contour surrounding $\sigma_{B}^{U}\left(\beta_{j}\right)$ in $U_{j}$, then for any $f \in A(U, X)$ we have

$$
v_{\beta}(f)=\frac{1}{(2 \pi i)^{n}} \int_{\Gamma_{1}} \ldots \int_{\Gamma_{n}} \beta_{1}\left(z_{1}\right)^{-1} \ldots \beta_{n}\left(z_{n}\right)^{-1} f(z) d z_{1} \ldots d z_{n}
$$

Proof. The assertion follows by induction from Theorem 3.8.

If $u=\left(u_{j k}\right)_{j, k=1}^{n} \subset A(U, B)$ is a matrix of commuting elements, we denote by det $u$ the determinant of this matrix.

Proposition 3.13. Let $U \subset C^{n+m}$ be an open set and $\beta=\left(\beta_{1}, \ldots, \beta_{n}\right) \subset A(U, B)$ a commuting system. Assume that $u=\left(u_{j k}\right)_{j, k=1}^{n}$ is a matrix of commuting elements of $A(U, B)$, which commute also with $\beta_{1}, \ldots, \beta_{n}$. Denote $\gamma_{j}=\sum_{k=1}^{n} u_{j k} \beta_{k}, \gamma=\left(\gamma_{1}, \ldots\right.$ $\left.\ldots, \gamma_{n}\right)$. If $\sigma_{B}^{U}(\beta)$ and $\sigma_{B}^{U}(\gamma)$ are both $C^{n}$-compact in $U$, then

$$
v_{y}(f)=v_{\beta}((\operatorname{det} u) f),
$$

for any $f \in A(U, X)$.

Proof. We define a map

$$
U_{\ni}(z, w) \rightarrow \tilde{u}(z, w) \in \mathscr{L}(\Lambda[s, X]),
$$

induced by the relations

$$
\begin{aligned}
\tilde{u}(z, w)\left(x s_{j_{1}} \wedge\right. & \left.\ldots \wedge s_{j_{p}}\right) \\
& =\sum_{k_{1}<\ldots<k_{p}}\left(\operatorname{det} u_{k_{k} j_{q}}\right)_{h, q}(z, w) x s_{k_{1}} \wedge \ldots \wedge s_{k_{p}} \quad(x \in X ; p \geqslant 1),
\end{aligned}
$$

and $\tilde{u}(z, w) x=x$. We then have

therefore

$$
\tilde{u}(z, w) \delta_{\beta(z, w)} \xi=\delta_{\gamma(z, w)} \tilde{u}(z, w) \xi \quad(\xi \in \Lambda[s, X]) ;
$$

$$
\left(\delta_{\gamma}+\bar{\partial}\right) \tilde{u} H(\beta) S f=\tilde{u}\left(\delta_{\beta}+\bar{\partial}\right) H(\beta) S f=\tilde{u} S f=S(\operatorname{det} u) f .
$$

On account of this equality we can consider the form

$$
\left(\delta_{\gamma}+\bar{\partial}\right)(\varphi H(\gamma) S(\operatorname{det} u) f-\varphi \tilde{u}(H(\beta) S f)),
$$

which is null in a neighbourhood of $\sigma_{B}^{U}(\beta) \cup \sigma_{B}^{U}(\gamma)$, where $\varphi$ has the properties from the definition (3.9) for both $\sigma_{B}^{U}(\beta)$ and $\sigma_{B}^{V}(\gamma)$, and its support is compact. The conclusion is then forced by Lemma 3.6 .

\section{The analytic functional calculus}

In this section we shall end the construction of the analytic functional calculus. The results of the previous section will be generally applied to the commuting systems of the form

$$
\beta(z)=\left(z_{1}-b_{1}, \ldots, z_{n}-b_{n}\right),
$$

where $b=\left(b_{1}, \ldots, b_{n}\right) \subset B$ is a commuting system.

THEOREM 4.1. Let $b=\left(b_{1}, \ldots, b_{n}\right)$ be a commuting system in $B$ and $U \subset C^{n}$ an open set such that $U \supset \sigma_{B}(b)$. Then the integral

$$
f(b)=\frac{1}{(2 \pi i)^{n}} \int_{\Sigma} f(z) M(z-b) \wedge d z_{1} \wedge \ldots \wedge d z_{n}
$$


defines a continuous unital algebra homomorphism from $A(U)$ into $B$ such that the image of the coordinate function $z_{j}$ is the element $b_{j}(j=1, \ldots, n)$, where $\Sigma$ is an admissible surface surrounding $\sigma_{B}(b)$ in $U$.

Proof. The linearity and the continuity of the map $f \rightarrow f(b)$ of $A(U)$ into $B$ follow from the definitions.

If $f$ is any complex polynomial in $z_{1}, \ldots, z_{n}$, then from Proposition 3.12, applied to the system $\left(z_{1}-b_{1}, \ldots, z_{n}-b_{n}\right)$, we obtain that the direct computation of $f(b)$ coincides with the value given by the formula (4.1). In particular, the image of 1 is the identity in $B$ and the image of the coordinate function $z_{j}$ is $b_{j}(j=1, \ldots, n)$.

The only thing to be proved is the multiplicativity of the map $f \rightarrow f(b)$. Let us consider two functions $f$ and $g$ in $A(U)$ and denote by $\beta_{j}(z)$ the element $z_{j}-b_{j}(j$ $=1, \ldots, n)$. Then $\beta=\left(\beta_{1}, \ldots, \beta_{n}\right) \subset A(U, B)$ is a commuting system and $\sigma_{B}^{U}(\beta)$ $=\sigma_{B}(b) \subset U$. Then we have by Theorem 3.8

$$
f(b) g(b)=v_{\beta}(f) v_{\beta}(g)=v_{(\beta, \beta)}(f g) .
$$

Consider now the matrix $u=\left(u_{j k}\right)_{j, k=1}^{2 n}$, where $u_{j k}=1$ if $j=k, u_{j k}=-1$ if $k=n+j$ and $u_{j k}=0$ otherwise. Note also that $\operatorname{det} u=1$. We shall apply Proposition 3.13. Namely, we transform the system

$$
\left(z_{1}-b_{1}, \ldots, z_{n}-b_{n}, w_{1}-b_{1}, \ldots, w_{n}-b_{n}\right)
$$

by the matrix $u$, and obtain the system

$$
\gamma(z, w)=\left(w_{1}-z_{1}, \ldots, w_{n}-z_{n}, z_{1}-b_{1}, \ldots, z_{n}-b_{n}\right) .
$$

We infer that $v_{(\beta, \beta)}(f g)=v_{\gamma}(f g)$. We shall apply once more Theorem 3.8. If $\varrho(z, w)$ $=z-w$, we have, by interchanging $z$ with $w$,

$$
v_{\gamma}(f g)=v_{(\varrho, \beta)}(f g)=v_{\beta}\left(v_{e_{w}}(f) g(w)\right)=v_{\beta}(f g)=(f g)(b),
$$

since $\nu_{o w}(f)=f(w)$, by the formula (3.1), and this finishes the proof.

Theorem 4.1 is a version of the well known result of Shilov-Arens-Calderón concerning the analytic functional calculus in Banach algebras [2]. This result has been considerably extended by J. L. Taylor [10]. As we have seen, in the case of $C^{*}$-algebras the analytic functional calculus can be given by the canonical formula (4.1).

COROLLARY 4.2. For any commuting system $b=\left(b_{1}, \ldots, b_{n}\right) \subset B$ there exists a unital algebra homomorphism from the algebra of germs of analytic functions in neighbourhoods of $\sigma_{B}(b)$ into $B$ such that the image of the germ of the coordinate function $z_{j}$ is $b_{j}(j=1, \ldots, n)$. Moreover, this homomorphism has values in the double commutant of the system $b$ in $B$.

Proof. The assertion follows from Lemma 3.6, Proposition 3.9 and Theorem 4.1.

THEOREM 4.3. Let $b=\left(b_{1}, \ldots, b_{n}\right)$ be a commuting system in $B, U \subset C^{n} a$ polydomain such that $U \supset \sigma_{B}(b)$ and $f=\left(f_{1}, \ldots, f_{m}\right)$ a system in $A(U)$. If $c_{j}=f_{j}(b)$ $(j=1, \ldots, m)$ and define $c=\left(c_{1}, \ldots, c_{m}\right)$, then $\sigma_{B}(c)=f\left(\sigma_{B}(b)\right)$
Proof. Let us denote by $A$ the double commutant of the system $b$ in $B$. It will be enough to prove that for any $g \in A(U)$ and every $\gamma \in \Gamma(A ; B)$ we have $\gamma(g(b))$ $=g(\gamma(b))$. We shall use an induction argument. For $n=1$ the assertion is well known [2] (and it can be easily proved). Define $b^{\prime}=\left(b_{1}, \ldots, b_{n}, b_{n+1}\right)=\left(b, b_{n+1}\right)$ and take $g^{\prime} \in A\left(U^{\prime}\right)$, where $U^{\prime} \supset \sigma_{B}\left(b^{\prime}\right)$, Then by Theorem 3.8 and Lemma 3.11 we can write

$$
g^{\prime}\left(b^{\prime}\right)=\frac{1}{2 \pi i} \int_{\Gamma}\left(w-b_{n+1}\right)^{-1} g_{w}^{\prime}(b) d w,
$$

where $T$ is an admissible contour surrounding $\sigma_{B}\left(b_{n+1}\right)$ in the projection of $U^{\prime}$ onto the last coordinate. Then for any $\gamma \in \Gamma(A ; B)$ we have, by the induction hypothesis,

$$
\gamma\left(g^{\prime}\left(b^{\prime}\right)\right)=\frac{1}{2 \pi i} \int_{\Gamma}\left(w-\gamma\left(b_{n+1}\right)\right)^{-1} g_{w}^{\prime}(\gamma(b)) d w=g^{\prime}\left(\gamma(b), \gamma\left(b_{n+1}\right)\right)=g^{\prime}\left(\gamma\left(b^{\prime}\right)\right) \text {. }
$$

This result is a variant of the spectral mapping theorem [2] (see [10] for more general conditions).

\section{References}

[1] S. B o c h n e r, Analytic and meromorphic continuation by means of Green's formula, Ann. of Math. 44 (1943), 652-673.

[2] N. B o u r b a ki, Théories spectrales, Chapitres 1-2, Hermann, Paris 1967.

[3] J. B u n c e and N. S a li n a s, Completely positive maps on $C^{*}$-algebras and the left matricial spectra of an operator, Duke Math. Journ. 43 (1976), 747-774.

[4] J. D ixmi er, Les $C^{*}$-algebres et leurs représentations, Gauthier-Villars, Paris 1969.

[5] N. D unf ord and J. S chwart z, Linear operators, Part I, Interscience Publishers, New York 1958.

[6] E. M a $\mathrm{r}$ i i $\mathrm{e} 11 \mathrm{i}$, Alcuni teoremi integrali per le funzioni analitiche di più variabili complesse, Memor. Acad. Ital. 9 (1938).

[7] F. Norguet, Introduction aux fonctions de plusieurs variables complexes: représentations intégrales, Lecture Notes in Math., 409, Springer-Verlag, 1974.

[8] S. S a k a i, $C^{*}$-algebras and $W^{*}$-algebras, Springer-Verlag, New York 1971.

[9] J. L. T a y 1 o r, A joint spectrum for several commuting operators, J. Funct. Analysis 6 (1970), 172-191.

[10] - The analytic functional calculus for several commuting operators, Acta Math. 125 (1970), $1-38$.

[11] F.-H. V a sil e scu, A characterization of the joint spectrum in Hilbert spaces, Rev. Roum Math. Pures Appl. 22 (1977), 1003-1009.

12] -, Analytic functional calculus and Martinelli's formula, Communication presented at the III Romanian-Finnish Seminar on Complex Analysis, Bucharest 1976.

[13] - Multi-dimensional analytic functional calculus (Romanian), The Publishing House of the Academy, Bucharest 1979.

[14] -, A Martinelli type formula for the analytic finctional calculus, Rev. Roum. Math. Pures Appl. 23(1978), 1587-1605.

Presented to the semester

Spectral Theory

September 23-December 16, 1977 